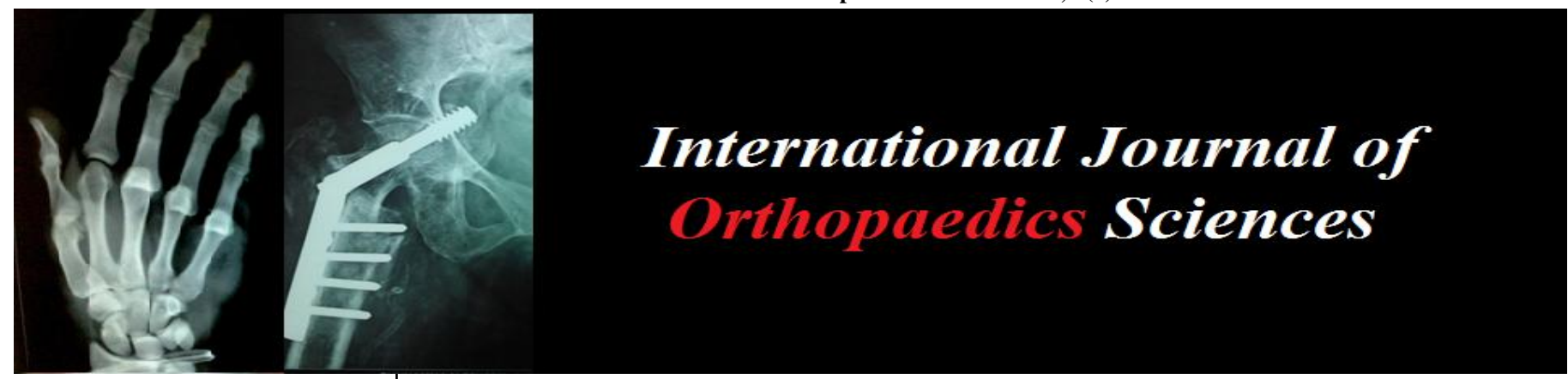

ISSN: $2395-1958$

IJOS 2017; 3(4): 634-639

(C) 2017 IJOS

www.orthopaper.com

Received: 29-08-2017

Accepted: 30-09-2017

Dr. Vishwas Phadke

Assistant Professor in

Orthopaedics, Bharati

Vidyapeeth Deemed University

Medical College, Sangli,

Maharashtra, India

Dr. Anil Khandekar

Associate Professor in

Orthopaedics, Terna Medical

College, Navi Mumbai,

Maharashtra, India

Dr. Nagesh Naik

Professor in Orthopaedics, Bharati Vidyapeeth Deemed

University Medical College,

Sangli, Maharashtra, India

Correspondence

Dr.Vishwas Phadke

Assistant Professor in

Orthopaedics, Bharati

Vidyapeeth Deemed University

Medical College, Sangli,

Maharashtra, India

\section{Chondrosarcoma of bone: A retrospective study of surgical management of 30 cases}

\author{
Dr. Vishwas Phadke, Dr. Anil Khandekar and Dr. Nagesh Naik
}

DOI: https://doi.org/10.22271/ortho.2017.v3.i4i.90

\section{Abstract}

Background: Chondrosarcomas are tumors that predominantly consist of cartilage and account for approximately $25 \%$ of all bone sarcomas. These tumors are relatively resistant to chemo and radiotherapy. They are classified into conventional Chondrosarcomas, de-differentiated Chondrosarcoma, mesenchymal Chondrosarcoma, juxtracortical Chondrosarcoma and secondary Chondrosarcoma on the basis of histopathology and whether or not they have occurred in a previously benign cartilaginous lesion. They are further subdivided into low grade, intermediate grade and high grade Chondrosarcoma on the basis of cellularity, atypia and pleomorphism. The clinical features of Chondrosarcoma include dull aching pain especially during night time, neurological symptoms (due to nerve involvement) and pathological fractures. Diagnosis is done by imaging and histopathology. Surgery is the primary treatment and consists of complete and wide excision whenever possible. Chemo and radiotherapy has got a limited role. We conducted this retrospective study of 30 patients with Chondrosarcoma treated with uniform surgical protocol at our institution.

Materials and Methods: After obtaining approval from institutional ethical committee we conducted a retrospective study of 30 patients diagnosed with chondrosarcoma. Since Chondrosarcomas are rare we searched for the patients records diagnosed with Chondrosarcoma and details were studied from case papers and patient examination during follow up. All patients had undergone surgical treatment of primary tumors. The various surgeries done in these patients included wide excision, Tikhoff-Linberg procedures, wide chest wall resections and hemipelvectomies depending upon the site and size of the tumors. Minimum follow up was 24 months. The data was tabulated and analyzed using SPSS 16.0 version software.

Results: This study consisted of 30 patients having been diagnosed with Chondrosarcoma. There were 18 men and 12 women with a mean age of 34 years (range $18-50$ years). The most common clinical features these patients presented with were swelling $(76.66 \%)$ followed by pain $(66.66 \%)$, neurological symptoms due to nerve involvement $(16.66 \%)$, fumigating skin lesion $(13.33 \%)$ and pathological fractures $(6.66 \%)$. Patients were diagnosed on the basis of Imaging (XRAY/CT /MRI) and biopsy followed by histopathological examination. Most common bones involved were femur $(30 \%)$ followed by pelvic bones $(26.67 \%)$ and humerus (13.33\%). Various surgical procedures done included complete excision in 15 patients $(50 \%)$ followed by Likhoff-Linberg procedure $(20 \%)$, chest wall resections $(16.66 \%)$ and hemipelvectomy (13.33\%). 9 patients were lost to follow up. Out of remaining 21 patients 15 patients $(50 \%)$ remained recurrence free during a follow up period of 24 months while 6 patients $(20 \%)$ presented with either localized recurrence or metastasis.

Conclusions : Chondrosarcomas are heterogeneous group of tumors ranging from low-grade to very aggressive, high-grade forms. In individuals presenting with localized disease wide surgical resection represents the primary treatment modality. Recurrences and metastatic disease is difficult to manage as chemotherapy is usually ineffective. Radiotherapy may be used in such cases as a palliation therapy.

Keywords: chondrosarcoma, tikhoff-linberg procedure, outcome, recurrence

\section{Introduction}

Chondrosarcoma is one of the cartilage forming tumors which is second most common solid bone tumor after osteosarcoma ${ }^{[1]}$. These tumors accounts for approximately $25 \%$ of primary malignant neoplasm of bones. Surgical resection has been the treatment of choice since these tumors are relatively resistant to chemo and radiotherapy ${ }^{[2]}$. Low grade and slowly growing Chondrosarcoma must be differentiated from benign cartilaginous lesions because of obvious prognostic and treatment implications. Chondrosarcomas are divided into low grade, 
intermediate grade and high grade on the basis of cellularity, atypia, and pleomorphism. Grading of these tumors is important as a low grade tumor rarely metastasize while a high grade tumor has high probability of local recurrence and metastasis. These neoplasms are divided into primary and secondary Chondrosarcoma on the basis of whether they have arisen denovo or from pre-existing cartilaginous lesions ${ }^{[3]}$. Though the exact cause of Chondrosarcoma is not known abnormalities localized to chromosomes $13 q 14$ and 17 p13 are associated with higher incidence of Chondrosarcoma [4]. Secondary Chondrosarcoma occurs in a patient having preexisting conditions like Maffucci syndrome, Ollier disease, osteochondromas, enchondroma and pagets disease [5]. Chondrosarcoma has a predilection for axial skeleton and most commonly affects femur, humerus pelvis or ribs and less commonly craniofacial bones or vertebrae ${ }^{[6]}$. Usually the patients present with either slowly or rapidly growing (depending upon whether it is low grade or high grade) swelling at the tumor site. Pain especially during night time is an important feature of Chondrosarcoma. Neurological manifestation may be the result of nerve involvement. Femoral nerve, sciatic nerve and lumbosacral plexus are commonly affected ${ }^{[7]}$. Though pain is a consistent symptom its nature initially is not severe hence various papers have reported the time from pain to diagnosis of Chondrosarcoma to be between 18 to 20 months ${ }^{[8]}$. The diagnosis is usually made on the basis of imaging which includes plain X-rays, Computed Tomography and Magnetic resonance imaging. Plain X-ray may show cartilagenous lesion with calcification along with cortical thickening and endosteal scalloping [9]. Higher the grade of tumor higher is the extent of bone destruction. Computed Tomographic scan may show matrix calcification, cortical breach and punctate calcification. The features are similar to as those seen on plain radiograph but are better appreciated on CT scan with a higher sensitivity for detection ${ }^{[10]}$. MRI may show tumor which is ISO to hypointense on T1 weighted images and hyperintense on T2 weighted images while on gradient images characteristic blooming can be well appreciated ${ }^{11}$. Nuclear imaging like bone scan is used to differentiate it from enchondroma which shows less intense uptake than Chondrosarcoma ${ }^{[12]}$. Further confirmation of diagnosis and staging depends upon histopathology which is crucial in the confirmation of diagnosis. Performing a proper biopsy is one of the crucial steps in diagnosis since Chondrosarcoma consist of different areas of different grades and it's absolutely essential that biopsy be taken from the area having most aggressive features [13]. Once the diagnosis is made surgery is the preferred treatment as these tumors are relatively resistant to chemo and radiotherapy. Complete and wide surgical excision of the tumors is the preferred method whenever it is feasible ${ }^{[14]}$. It is more feasible in appendicular skeleton than axial skeleton where a wide excision may not be possible. Radiotherapy and chemotherapy has got a very limited role in treatment but can be used as adjuvant treatment when complete excision is not possible or for the palliative treatment in patients with metastatic pain ${ }^{[15]}$. Regular follow up clinical examination and radiological investigations (XRAY, CT or Bone scan) is essential to know the local recurrence or distant metastasis. We conducted this retrospective study to know the demographic characteristics, treatment modalities and outcome in patients with Chondrosarcoma admitted to our institute.

\section{Materials and Methods}

This study was conducted at department of orthopedics of a medical college situated in an urban area. The study was duly approved by institutional ethical committee. Since Chondrosarcoma is a rare neoplastic disease hence we designed a retrospective study. The case papers (OPD and IPD) of all the patients diagnosed with Chondrosarcoma were studied in detail. The information which was recorded included demographic profile (age sex and address), history, clinical examination with a special emphasis on location of swelling and nature of pain, Reports of investigations like Complete blood count and other biochemical investigations, Imaging results (X-Ray, Computed Tomography, Magnetic Resonance Imaging and Bone Scan) and finally report of histopathology. Whenever available, additional clinical and diagnostic results were also noted. The treatment like type of surgery, adjuvant chemo or radiotherapy (if given) was also noted down. Follow up records of the treated patients were reviewed up to 24 months for recurrence of local disease or metastasis. Those patients who didn't come for any follow up visits were excluded from the study. Whenever needed and whenever it was feasible a direct telephonic communication was made with patients, pathologists, radiologists and nuclear medicine consultants or family members.

The results were studied using appropriate statistical methods. $\mathrm{P}<0.05$ was taken as statistically significant. Data analysis was carried out using SPSS16.0 version software. Microsoft word and excel were used for generating charts and graphs

\section{Inclusion Criteria}

1. All cases of Chondrosarcoma confirmed on radiology and histopathology were included in this study.

2. Age of the patient should be 18 years or more.

3. Surgically respectable disease.

4. Patients who attended follow up OPDs at least for 2 years.

\section{Exclusion Criteria}

1. Age less than 18 years.

2. Surgically unresectable tumors.

3. Patients who didn't come for follow up visits or when follow up records couldn't be obtained from case papers.

4. Patients presenting with metastasis.

\section{Results}

This was a retrospective study in which case records (OPD and IPD) of 30 patients diagnosed to have Chondrosarcoma were studied with a view to analyze the presenting complaints, clinical features, mode of treatment and outcomes if they met the inclusion criteria. Patients having any exclusion criteria were excluded from the study. There were 36 patient found to have been diagnosed with Chondrosarcoma during specified period. Out of these 36 patients 6 patients had one or more exclusion criteria and hence were excluded from the study. Remaining patients were included in this study. Out of 30 patients 18 were males and 12 were females with a $\mathrm{M}$ : F ratio being 1:0.6. 


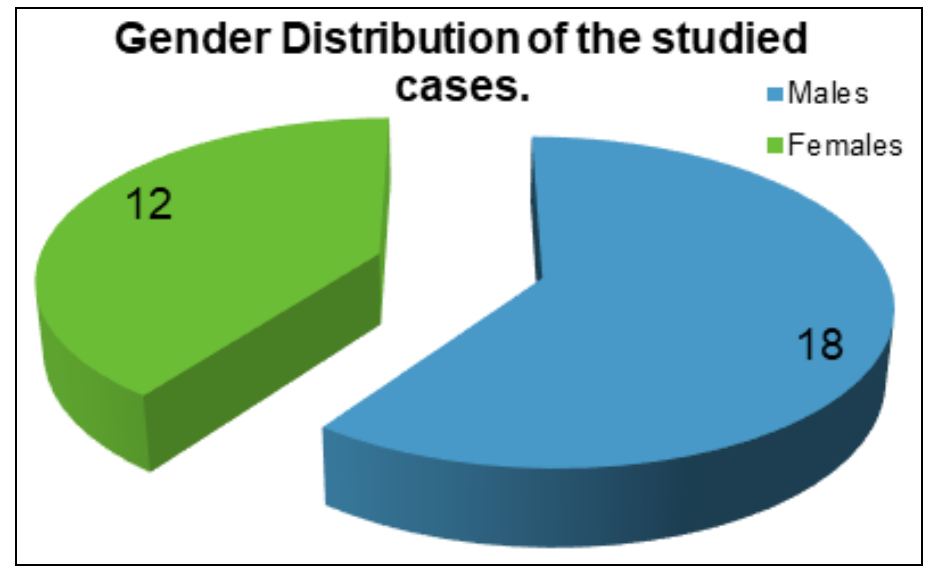

Fig 1: Gender Distribution of the studied cases

The analysis of age groups of the studied patients revealed that the most common age group affected was 31-40 years $(43.33 \%)$ followed by $40-50$ years $(36.66 \%), 21-30$ years
$(13.33 \%)$ and less than 20 years (6.66\%). Patients less than 18 years were excluded from the study since age more than 18 years was an inclusion criteria.

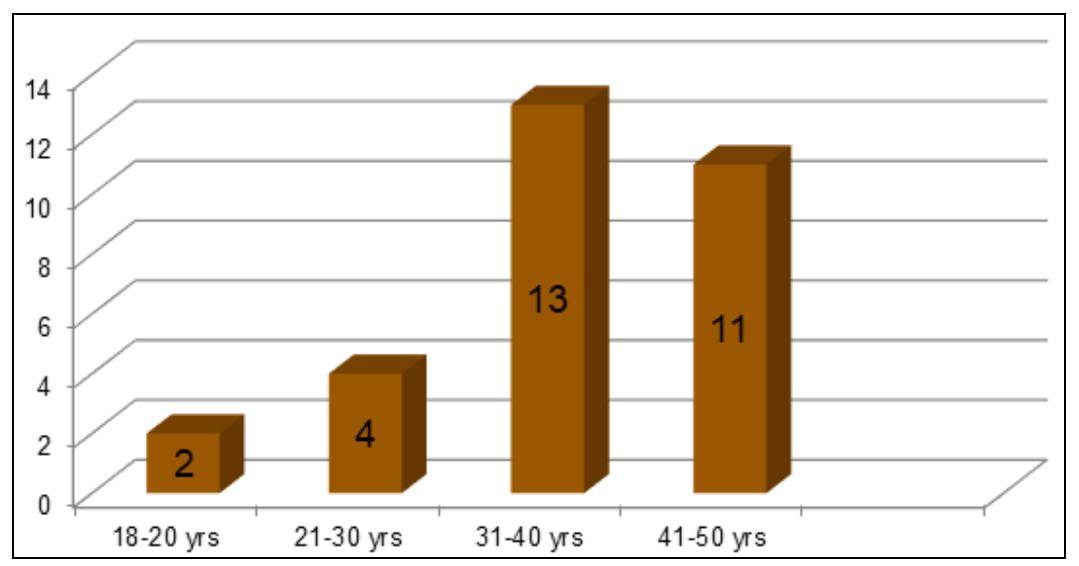

Fig 2: Age distribution of the studied cases.

The analysis of clinical features seen in these patients revealed that the most common sign was a gradually increasing swelling at the affected site which was present in $23(76.66 \%)$ patients followed by pain which was present in $20(66.66 \%)$ and neurological symptoms due to compression of neural tissue which was seen in 5 (16.66\%). 4 (13.33\%) patients presented with fungating skin lesions while $2(6.66 \%)$ patients presented with pathological fracture secondary to Chondrosarcoma.

Table 1: Signs and symptoms in the studied cases.

\begin{tabular}{|c|c|c|}
\hline Sign and Symtopms & No of patients & Percentage \\
\hline swelling at the affected site & 23 & $76.66 \%$ \\
\hline pain & 20 & $66.66 \%$ \\
\hline neurological symptoms & 5 & $16.66 \%$ \\
\hline Fungating Skin Lesions & 4 & 13.33 \\
\hline Pathological Fractures & 2 & $6.66 \%$ \\
\hline
\end{tabular}

The analysis of location of the tumors revealed that the most common site of primary tumor was femur $(30 \%)$ followed by pelvic bones $(26.67 \%)$, humerus $(13.33 \%)$, tibia $(10 \%)$, ribs $(6.67 \%)$, scapula $(6.67 \%)$, spine $(3.33 \%)$ and sternum $(3.33 \%)$.

Table 2: Location of the tumor in the studied cases.

\begin{tabular}{|c|c|c|}
\hline Location & No of patients & Percentage \\
\hline Femur & 9 & $30 \%$ \\
\hline Pelvic bones & 8 & $26.67 \%$, \\
\hline Humerus & 4 & $13.33 \%$ \\
\hline Tibia & 3 & $10 \%$ \\
\hline Ribs & 2 & $6.67 \%$ \\
\hline Scapula & 2 & $6.67 \%$ \\
\hline Spine & 1 & $3.33 \%$ \\
\hline Sternum & 1 & $3.33 \%$ \\
\hline Total & 30 & $100 \%$ \\
\hline
\end{tabular}


The imaging studies done in patients revealed calcified, eccentric osteolytic lesions on pain X-rays. Computed tomography showed calcified mass which was well defined with soft tissue extensions and local destruction. MRI showed lesions which were ISO to hypo intense on T1 weighted images and hyperintense on $\mathrm{T} 2$ weighted images while on gradient images characteristic blooming was well appreciated.

Table 3: Imaging findings in the studied cases.

\begin{tabular}{|c|c|}
\hline Imaging Modality & \multicolumn{1}{c|}{ Appearance } \\
\hline Plain Radiographs & Calcified, eccentric osteolytic lesions \\
\hline Computed Tomography & Calcified mass which was well defined with soft tissue extensions and local destruction. \\
\hline Magnetic Resonance Imaging & $\begin{array}{l}\text { Lesions which were ISO to hypo intense on T1 weighted images and hyperintense on T2 } \\
\text { weighted images while on gradient images characteristic blooming was well appreciated. }\end{array}$ \\
\hline
\end{tabular}

Histopathological examination and confirmation was done in all the cases. It demonstrated characteristic sheets of rounded or spindled mesenchymal cells with islands of hyaline cartilages. Majority of the tumors showed predominantly spindle cells in histopathological examination. Histological features also included ossification and calcification.

Since the primary treatment of Chondrosarcoma consists of wide and complete resection majority of patients underwent surgical excision whenever it was feasible. Various surgical procedures done in these patients included local surgical decompression in $15(50 \%)$ patients followed by TikhoffLinberg procedure 6 (20\%), chest wall resections 5 (16.66\%) and Hemipelvectomy which was done in $4(13.33 \%)$ patients.

Table 4: Surgical Procedures done in the studied cases.

\begin{tabular}{|c|c|c|}
\hline Surgical Procedure & No of Patients & Percentage \\
\hline Complete local excision & 15 & $50 \%$ \\
\hline Tikhoff-Linberg procedure & 6 & $20 \%$ \\
\hline Chest wall resections & 5 & $16.66 \%$ \\
\hline Hemipelvectomy & 4 & $13.33 \%$ \\
\hline
\end{tabular}

The analysis of follow up records of the patients (for at least 2 years) revealed that $9(30 \%)$ patients were lost to follow up. Out of remaining patients 15 patients (50\%) remained recurrence free during a follow up period of 24 months while 6 patients $(20 \%)$ presented with either localized recurrence or metastasis.

Table 5: Outcome of the studied cases over a follow up period of 2 years.

\begin{tabular}{|c|c|c|}
\hline Outcome & No of patients & Percentage \\
\hline Lost to Follow Up & 9 & $30 \%$ \\
\hline Disease free & 15 & 50 \\
\hline Localized recurrence & 4 & 13.33 \\
\hline Metastasis & 2 & 6.66 \\
\hline
\end{tabular}

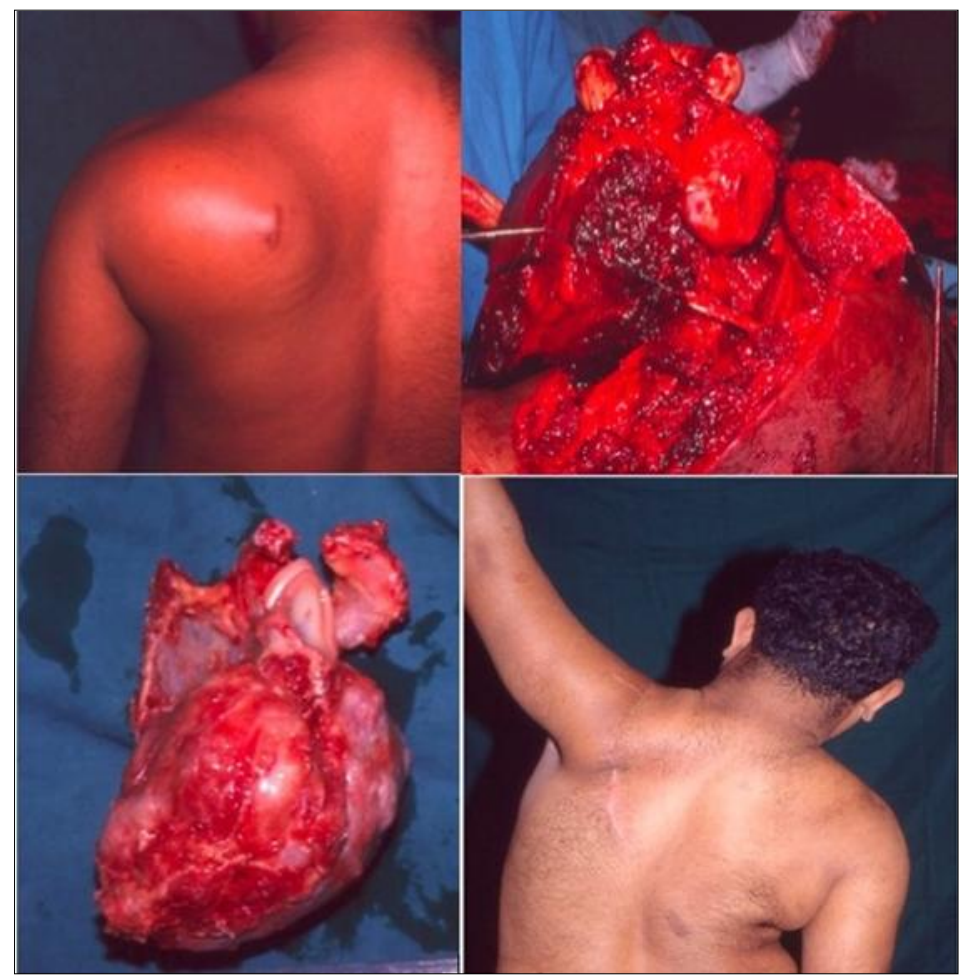

Fig 3: Chondrosarcoma of left Scapula treated by Tikhoff-Linberg Operation. Note excellent postoperative functional outcome. 


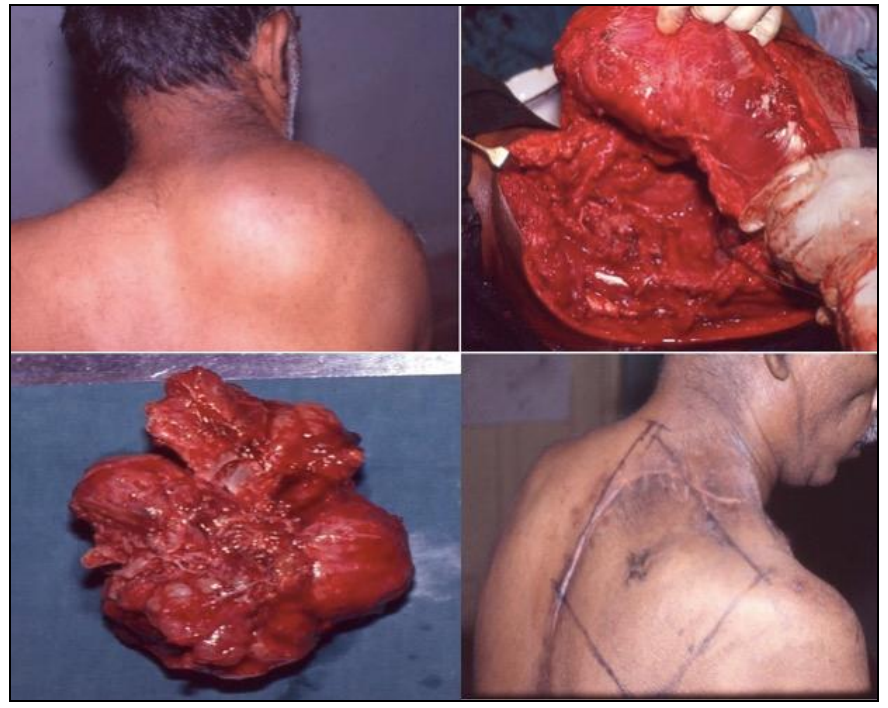

Fig 4: Chondrosarcoma of second Rib Treated by complete Excision.

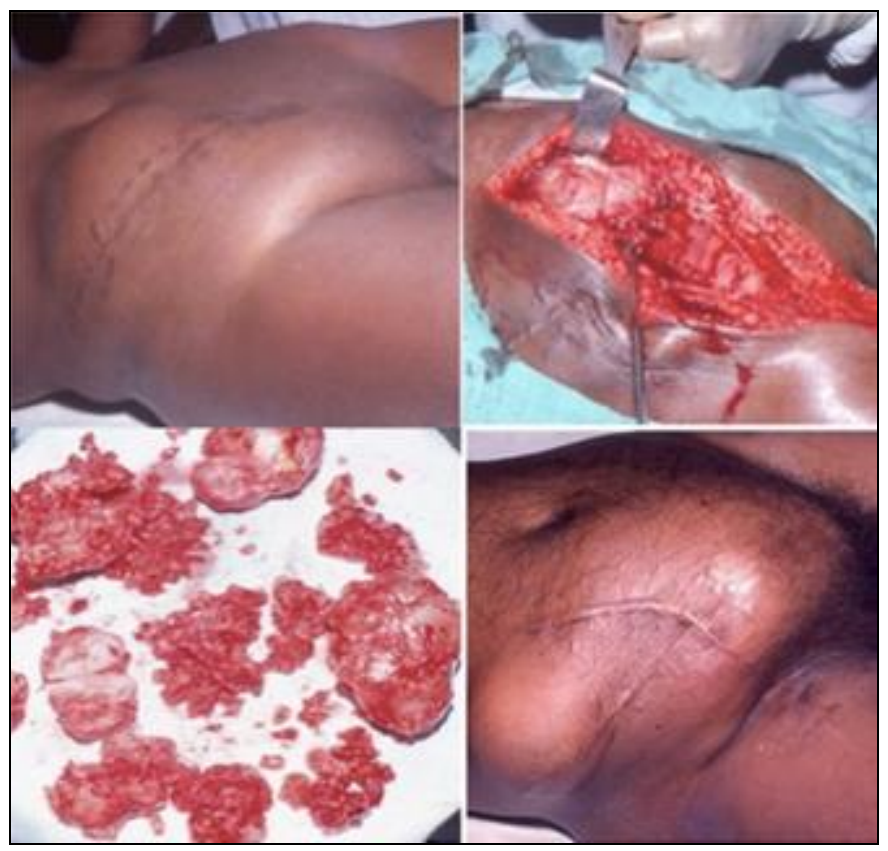

Fig 5: Chondrosarcoma of Pelvic bone treated by hemipelvectomy. Unfortunately there was a local recurrence after 1 year.

\section{Discussion}

Chondrosarcoma as discussed earlier is the second most common bone tumor only to be followed by ostesarcoma. Chondrosarcoma can affect any bone but have predilection of femur humerus and pelvic bones. Males are more commonly affected than females. Various studies have confirmed male preponderance for chondrosarcoma ${ }^{[16,17]}$. The exact cause of male preponderance for these tumors is not known. Chondrosarcoma is usually seen in patients in between the age of 30-70 years and is rare below 20 years. Our study also confirm this finding as majority of the patients in our study were found to be between the ages of 20 to 50 years of age and only 2 patients were seen below the age of 20 years. Similar findings were seen in the studies conducted by Gerhard M. Hobusch et al. and Welkerling $\mathrm{H}$ et al. ${ }^{[18,19]}$

The most common clinical presentation of Chondrosarcoma consist of local swelling which may increase slowly or rapidly depending upon the grade of tumor. A high grade tumor is expected to grow rapidly and chances of recurrence after excision and distant metastasis is more in high grade tumor.
Other symptoms may include pain, neurological involvement due to nerve entrapment and pathological fractures ${ }^{[20]}$.

The diagnosis mainly depends upon imaging studies and histopathological confirmation. The difficulty in diagnosis arises from the variable nature of the tumor which may consist of locally destructive osteolytic lesions, calcified lesions and cartilaginous components. Advances in imaging techniques with more frequent use of computed tomography and magnetic resonance imaging has made the diagnosis of these tumors relatively easy. On plain radiographs these tumors may appear as lytic lesions with intralesional calcifications, endosteal scalloping, periosteal reaction and cortical remodeling. While on computed tomography the features are similar to plain X-ray but they are better appreciated. Heterogeneous contrast enhancement may be seen in cases where contrast enhanced CT has been done. On Magnetic resonance imaging the lesion is characteristically iso to hypointense on T1 weighted images and hyper intense on $\mathrm{T} 2$ weighted images with characteristics blooming on gradient images ${ }^{[21]}$. The confirmation of the diagnosis by histopathology is necessary not only for the diagnosis but also for the grading of tumors. While a low grade tumor is expected to have low chances of recurrence a high grade tumor is more likely to recur and hence the follow up can be planned depending upon the grade of tumor on histopathology [22].

The treatment mainly consists of complete excision of tumor as these tumors are fairly resistant to chemo and radiotherapy. The complete resection of Chondrosarcoma is reported to have an excellent prognosis is cases of low grade tumors and reported to have a 10 years survival in more than $50 \%$ patients. High grade Chondrosarcoma may have a less survival period because of increased chances for recurrence and metastatic disease ${ }^{[23]}$. Inadequate removal of margins is associated with increased chances of local recurrence. This is a very important fact to be considered while during surgeries in pelvic region where complete excision may be difficult. Incomplete excision may lead to local recurrence and this possibility is more while treating pelvic Chondrosarcoma like we have seen in our study. Pring ME et al. have also found similar findings in their study of 64 cases with Chondrosarcoma of pelvis ${ }^{[24]}$.

Follow up of the patients with Chondrosarcoma who have undergone complete excision is one of the important part of overall management. There are 2 very important factors which need to be considered for follow up. One is histological grade of the tumor since the high grade tumors are more likely to recur and hence a more frequent and close follow up is required. Another factor which needs to be considered is the site of the tumor. Pelvic Chondrosarcoma is having higher chances of recurrence because of the difficulty in complete excision. The role of chemo and radiotherapy is limited to the treatment of patients with metastasis and for palliative care [25].

\section{Conclusion}

Chondrosarcoma is the second most common malignant bone tumor. The diagnosis is usually done by imaging and histopathology. Treatment is by complete excision. Outcome usually depends upon the grade of tumor and whether complete excision of tumor was possible or not. Chemo and radiotherapy is usually reserved for metastatic disease and for palliation. 


\section{Conflict of interest}

None

\section{References}

1. von Eisenhart-Rothe R, Toepfer A, Salzmann M, Schauwecker J, Gollwitzer H, Rechl H. Primary malignant bone tumors. Orthopade. 2011; 40(12):112142.

2. Dai X, Ma W, He X, Jha RK. Review of therapeutic strategies for osteosarcoma, chondrosarcoma, and Ewing's sarcoma. Medical Science Monitor: International Medical Journal of Experimental and Clinical Research. 2011; 17(8):RA177-RA190.

3. Gelderblom H, Hogendoorn PC, Dijkstra SD, van Rijswijk CS, Krol AD, Taminiau AH et al. The clinical approach towards chondrosarcoma. Oncologist. 2008; 13(3):320-9.

4. Bovée JV, Cleton-Jansen AM, Kuipers-Dijkshoorn NJ, van den Broek LJ, Taminiau AH, Cornelisse CJ et al. Loss of heterozygosity and DNA ploidy point to a diverging genetic mechanism in the origin of peripheral and central chondrosarcoma. Genes Chromosomes Cancer. 1999; 26(3):237-46.

5. Bovée JV. Multiple osteochondromas. Orphanet Journal of Rare Diseases, 2008; 3:3.

6. Teo HEL, Peh WCG. Primary bone tumors of adulthood. Cancer Imaging. 2004; 4(2):74-83.

7. Katonis $\mathrm{P}$, Alpantaki K, Michail K et al. Spinal Chondrosarcoma: A Review. Sarcoma, 2011, 378957.

8. Young PS, Bell SW, MacDuff EM, Mahendra A. Primary Osseous Tumors of the Hind foot: Why the Delay in Diagnosis and Should We Be Concerned? Clinical Orthopaedics and Related Research. 2013; 471(3):871877.

9. Ollivier L, Vanel D, Leclère J. Imaging of chondrosarcomas. Cancer Imaging. 2004; 4(1):36-38.

10. Kenney PJ, Gilula LA, Murphy WA. The use of computed tomography to distinguish osteochondroma and chondrosarcoma. Radiology. 1981; 139(1):129-37.

11. Varma DG, Ayala AG, Carrasco CH, Guo SQ, Kumar R, Edeiken J Chondrosarcoma: MR imaging with pathologic correlation. Radiographics. 1992; 12(4):687-704.

12. Brenner W, Conrad EU, Eary JF. FDG PET imaging for grading and prediction of outcome in chondrosarcoma patients. Eur J Nucl Med Mol Imaging. 2004 ;31(2):18995.

13. Rinas AC, Ward WG, Kilpatrick SE. Potential sampling error in fine needleaspiration biopsy of dedifferentiated chondrosarcoma: a report of 4 cases. Acta Cytol. 2005; 49(5):554-9.

14. Donati D, Colangeli S, Colangeli M, Di Bella C, Bertoni F. Surgical Treatment of Central Chondrosarcoma. Clinical Orthopaedics and Related Research. 2010; 468(2):581-589.

15. Gulia A, Byregowda S, Panda PK. Palliative Care in Musculoskeletal Oncology. Indian Journal of Palliative Care. 2016; 22(3):244-251.

16. Giuffrida AY, Burgueno JE, Koniaris LG, Gutierrez JC, Duncan R, Scully et al. Chondrosarcoma in the United States 1973 to 2003: an analysis of 2890 cases from the SEER database. SPJ Bone Joint Surg Am. 2009; 91(5):1063-72.

17. Bindiganavile S, Han I, Yun JY, Kim H-S. Long-term Outcome of Chondrosarcoma: A Single Institutional Experience. Cancer Research and Treatment: Official
Journal of Korean Cancer Association. 2015; 47(4):897903.

18. Hobusch GM, Tiefenboeck TM, Patsch J, Krall C, Holzer G. Do Patients after Chondrosarcoma Treatment Have Age-appropriate Bone Mineral Density in the Long Term? Clinical Orthopaedics and Related Research. 2016; 474(6):1508-1515.

19. Welkerling H, Dreyer T, Delling G. Morphological typing of chondrosarcoma: a study of 94 cases. Virchows Arch A Pathol Anat Histopathol. 1991; 418(5):419-25.

20. Yoo HJ, Hong SH, Choi JY, Moon KC, Kim HS, Choi JA et al. Differentiating high-grade from low-grade chondrosarcoma with MR imaging. Eur Radiol. 2009; 19(12):3008-14.

21. Welkerling H, Werner M, Delling G. Histologic grading of chondrosarcoma. Aqualitative and quantitative analysis of 74 cases of the Hamburg bone tumorregister. Pathologe. 1996; 17(1):18-25.

22. Andreou D, Ruppin S, Fehlberg S, Pink D, Werner M, Tunn PU et al. Survival and prognostic factors in chondrosarcoma: Results in 115 patients with long-term follow-up. Acta Orthopaedica. 2011; 82(6):749-755.

23. Weber KL, Pring ME, Sim FH. Treatment and outcome of recurrent pelvic chondrosarcoma. Clin Orthop Relat Res., 2002; (397):19-28.

24. Pring ME, Weber KL, Unni KK, Sim FH. Chondrosarcoma of the pelvis. A review of sixty-four cases. J Bone Joint Surg Am. 2001; 83A:1630-42.

25. Italiano A, Mir O, Cioffi A et al. Advanced chondrosarcomas: role of chemotherapy and Survival. Annals of Oncology. 2013; 24(11):2916-2922. 